\title{
Caffeine delays habituation of the human acoustic startle reflex
}

\author{
EDWARD J. SCHICATANO and TERRY D. BLUMENTHAL \\ Wake Forest University, Winston-Salem, North Carolina
}

\begin{abstract}
The acoustic startle reflex has proved to be an excellent system for studying habituation in mammals. In animal studies, startle habituation has been found to be sensitive to various pharmacological manipulations. The present experiments were designed to determine whether caffeine $(4 \mathrm{mg} / \mathrm{kg})$ modified startle habituation in low and high caffeine users. Human eyeblink responses were measured in a startle habituation paradigm in which 30 trials of $85-\mathrm{dB}$ broadband noise stimuli with a duration of $50 \mathrm{msec}$ and a rise time of $.1 \mathrm{msec}$ were presented. Caffeine delayed the habituation of startle amplitude in both low and high users and produced significant dishabituation in low users. These findings indicate that caffeine disrupts early sensory filtering.
\end{abstract}

Habituation, an example of neural plasticity (Groves \& Thompson, 1970), is the simplest form of learning. This ubiquitous type of learning is recognized as a function of a system that requires minimal higher cortical processing. Nevertheless, the ability to habituate is essential to normal information processing and selective attention (Geyer \& Braff, 1987). The acoustic startle reflex has proved to be an excellent system for studying habituation in mammals (Braff \& Geyer, 1990; Davis \& Sheard, 1974). In humans, the acoustic startle reflex is elicited by a sudden, rapid onset stimulus with an intensity greater than $50 \mathrm{~dB}$ (Blumenthal, 1988; Blumenthal \& Goode, 1991), and the eyeblink component of this reflex may be elicited as early as $20 \mathrm{msec}$ following stimulus onset (Blumenthal \& Berg, 1986). Startle can occur in response to stimuli in several sensory modalities; it is sensitive to various stimulus parameters, such as intensity or duration; and it has been used as a model to assess the effects of drugs on sensorimotor reactivity (Geyer \& Braff, 1987). Furthermore, startle is indicative of rapid sensory information processing, and it can be used as a means for investigating nervous system functioning (Anthony, 1985).

The sensitivity of startle habituation to pharmacological manipulations has been reliably demonstrated in animal studies (Davis, 1980, 1984), although few human studies of this kind exist. Caffeine is the most widely used psychoactive substance (Snyder \& Sklar, 1984), and its effects have been studied in a variety of cognitive /behavioral tasks (Lieberman, Wurtman, Emde, Roberts, \&

Portions of these data were presented at the 32nd annual meeting of the Society for Psychophysiological Research, October 1992, in San Diego, California. E.J.S. is in the Neuroscience Program at Wake Forest. Correspondence should be addressed to T. D. Blumenthal, Department of Psychology, Wake Forest University, Box 7778 Reynolda Station, Winston-Salem, NC 27109.
Coviela, 1987). Caffeine has been found to improve motor performance in a visual and auditory vigilance task (Lieberman et al., 1987). Another experiment demonstrated that caffeine improved performance on a sustained attention task (A. P. Smith, Rusted, EatonWilliams, Savory, \& Leathwood, 1990). Although caffeine has a pronounced effect on several cognitive and motor tasks, its effect on brainstem-controlled behavior is not clear.

B. D. Smith, Rafferty, Lindgren, D. A. Smith, and Nespor (1991) have shown that caffeine ingestion enhances physiological responses to arousing external stimuli. Using a measure of skin conductance, these researchers demonstrated that caffeine slowed habituation to auditory stimuli. This reduced rate of habituation was more pronounced in high users of caffeine than in low users. Thus, caffeine appears to modulate habituation of skin conductance responses.

In rats, caffeine doses of up to $50 \mathrm{mg} / \mathrm{kg}$ produced a substantial increase in tactile startle amplitude (Ward, Pollare, \& Geyer, 1981). However, Manspeizer and Blumenthal (1986) reported no significant caffeine effect (doses were 2 or $4 \mathrm{mg} / \mathrm{kg}$ ) on acoustic startle in humans when investigating startle stimuli of different durations. It should be noted that, in Manspeizer and Blumenthal's study, the startle response was averaged over the entire session. Therefore, a specific effect observed at either the beginning or the end of the session might not have been observable when all trials were averaged together. In a habituation study, the entire session is broken down into trial blocks, enabling any changes that occur throughout the test session to be revealed.

In the present experiment, we investigated the effect of caffeine on startle habituation. In order to avoid any confounding effects that might be due to caffeine withdrawal or tolerance, noncaffeine users or low users were chosen as subjects. Thus, the acute effects of caffeine on startle habituation were investigated in the first experiment. 
Drug history influences the effects that a drug may have on an individual (Nehlig, Daval, Boyet, \& Vert, 1986). Abstinence from caffeine typically results in caffeine withdrawal, which is evidenced by a constellation of physical and psychological symptoms (Griffiths \& Woodson, 1988). In humans, peak withdrawal symptoms occur after $20-48 \mathrm{~h}$ of abstinence from caffeine (Griffiths, Bigelow, \& Liebson, 1986). To this end, persons with a history of high caffeine intake were chosen as subjects to assess the effects of caffeine withdrawal on startle habituation in Experiment 2.

Lastly, the effects of caffeine on the recovery of the habituated response was evaluated. Recovery of the habituated response to the original startle stimulus (S1) was examined by presenting several trials of a stimulus that was different from $\mathrm{S} 1$ and then measuring the response to subsequent re-presentations of S1. This kind of response recovery, called dishabituation, represents another form of plasticity; it can be tested following the occurrence of habituation. Hence, the effects of caffeine on two different types of plasticity-habituation and dishabituation-were tested in Experiments 1 and 2.

\section{EXPERIMENT 1}

\section{Method}

Subjects. Nine subjects ( 2 males and 7 females; average age $=$ 18 years, 8 months) participated; they were chosen from an undergraduate introductory psychology class on the basis of their responses to a 34 -item questionnaire indicating low or no caffeine intake (i.e., less than $100 \mathrm{mg}$ of caffeine per week). For example, when considering the wide range of caffeine-containing substances, these subjects reported consuming only 1 or 2 sodas per week at most.

Stimuli. The startle stimuli were $85-\mathrm{dB}$ (re: $20 \mu \mathrm{PA}$; A scale) broadband noise $(20 \mathrm{~Hz}-20 \mathrm{kHz})$, with a duration of $50 \mathrm{msec}$ and a rise time of $.1 \mathrm{msec}$. A $75-\mathrm{dB}$ (A scale) $1000-\mathrm{Hz}$ tone with a rise time of $10 \mathrm{msec}$ and a duration of $50 \mathrm{msec}$ was used to elicit dishabituation. The interstimulus interval (ISI) varied randomly from 20 to $30 \mathrm{sec}$ (average ISI $=25 \mathrm{sec}$ ).

Materials. The caffeine solution consisted of $100 \%$ pure anhydrous caffeine (Carolina Biological Supply) dissolved in distilled water and mixed with Tang orange drink. The caffeine dose was $4 \mathrm{mg} / \mathrm{kg}$ for each subject. The placebo solution consisted of flat tonic water mixed with Tang orange drink. Since flat tonic water has a bitter taste, the placebo and caffeine drinks tasted similar. The fluid volume per body weight was identical for both placebo and drug solutions.

Apparatus. The stimuli were produced with a Coulbourn S8102 noise generator and a Coulbourn S81-06 signal generator, gated through a Coulbourn S84-04 electronic switch, a Coulbourn S8224 audio amplifier, a Yamaha M-35 stereo amplifier, and presented to the subjects through an Onkyo S-30 loudspeaker. The stimulus intensity was calibrated by presenting a continuous stimulus from the loudspeaker to a Quest Electronics 215 (A scale) sound level meter held at the location of the subject's head.

Reflex eyeblink responses were assessed from periorbital EMG activity collected through miniature Sensormedics biopotential electrodes $(\mathrm{Ag} / \mathrm{AgCl})$ filled with conducting paste. The EMG signal was amplified by a Coulbourn S75-01 high-gain bioamplifier/coupler with filters passing $90-250 \mathrm{~Hz}$. The amplifier output was sent to a Coulbourn S76-01 contour-following integrator with a 10-msec time constant. The output of the integrator was digitally sampled by a MacPacq MP10 interface every millisecond for
$1,000 \mathrm{msec}$ after startle stimulus onset. The responses were viewed and stored on a Macintosh SE microcom puter.

The dependent variables consisted of response amplitude, latency, and probability measured by EMG recordings from the orbicularis oculi muscle below the left eye. Response amplitude was measured as the difference between response onset and peak in arbitrary units; response latency was measured as the time from stimulus onset to response onset; and response probability was measured as the percentage of trials on which a response was elicited, given that a response could have been recorded. When scoring data, only responses beginning within $20-100 \mathrm{msec}$ after startle stimulus onset were included, to eliminate nonreflexive responses from the data.

Procedure. The use of students from an introductory psychology class was approved by the Wake Forest University Institutional Review Board. The subjects were asked to refrain from consuming caffeine in any form for $12 \mathrm{~h}$ prior to each experimental session. When each subject arrived, he/she was first weighed and was then asked to read and sign an informed consent form and to fill out a background questionnaire. All 9 subjects were given caffeine and placebo solutions on separate days, with at least 1 week separating the testing sessions. Five subjects received the caffeine condition during the first testing session and the placebo condition during the second testing session. The remaining 4 subjects were presented with these conditions in the opposite order.

Each subject received the caffeine solution or placebo solution, which was given in a double-blind fashion. Caffeine is rapidly absorbed, reaching maximal plasma levels within about 30 min after oral administration in humans (Griffiths \& Woodson, 1988). Twenty minutes after ingestion of the solution, the experimenter began the electrode preparation procedure. The experimenter cleaned the area just below the subject's left eye with a cotton swab dipped in alcohol and attached two electrodes, one below the center of the eye, and the other immediately temporal to the first, as close to the orbital ridge as possible. A ground electrode was placed on the medial surface of the left forearm. The subjects were seated in a chair located approximately $1.5 \mathrm{~m}$ in front of a loudspeaker and were presented with the first acoustic stimulus $30 \mathrm{~min}$ after they had ingested the mixed solution. Each subject was exposed to 42 trials: 30 broadband noise trials, followed by 6 tone trials and then 6 trials of the original broadband noise stimulus. The entire session lasted approximately $20 \mathrm{~min}$.

Data analysis. The startle response amplitudes, latencies, and probabilities for each subject were first condensed into 10 blocks of 3 consecutive trials each. Habituation was assessed in a trend analysis of the first 10-trial blocks. Slope comparisons measuring differences in the amount of habituation between the drug and placebo conditions were analyzed by BMDP2V orthogonal trend comparisons. Post hoc tests comparing individual trial blocks were analyzed in a two-way analysis of variance (ANOVA; BMDP4V). A two-way ANOVA was also used to determine where habituation reached asymptote in the drug and placebo conditions. Peak habituation was considered to occur on the last trial block after which no later trial blocks were significantly different. In subsequent orthogonal trend analysis, only trial blocks up to the point at which habituation reached asymptote were included. This subsequent analysis provided a more specific comparison of the habituation curves by excluding later trials on which the curves were asymptotic. Response amplitude in the first trial block was compared between drug conditions to determine differences in startle reactivity.

The tone trials in this experiment were designed to produce dishabituation, which would be made evident by a larger response for Trial Block 13 than for Trial Block 10. These data were analyzed in a mixed design ANOVA with the drug-placebo conditions as the between-subjects variable, and the average trial block comparisons as the within-subjects variable. This specific trial block comparison involves the average response to the last three noise 
presentations on the first 30 trials, and the average response to the first three noise presentations following the tone stimuli.

\section{Results}

No significant main effect of drug condition was found for response amplitude, latency, or probability. An orthogonal trend analysis of response amplitude indicated a significant trial block effect when 10 trial blocks were analyzed $[F(1,16)=21.25, p<.001]$, and there was no significant drug $\times$ trial block interaction. Post hoc ANOVAs revealed no significant decreases in response amplitude following Trial Block 3 in the placebo condition and following Trial Block 5 in the caffeine condition. These results demonstrated that habituation reached asymptote on these trial blocks for the placebo and caffeine conditions. An orthogonal trend analysis of the first 5 trial blocks revealed a drug $\times$ trial block interaction $[F(1,16)=4.93, p<.05]$. (See Figure 1.)

Follow-up pairwise comparisons were conducted to assess habituation differences between the caffeine and placebo conditions. Response differences between Trial Blocks 1 and 2 were significant in the placebo but not in the caffeine condition $[F(1,16)=5.00, p<.05]$. Likewise, response differences between Trial Blocks 1 and 3 were significant in the placebo but not in the caffeine condition $[F(1,16)=4.69, p<.05]$. These comparisons illustrated a significant response decrement (habituation) as early as Trial Block 2 (Trials 4-6) for the placebo condition. The remaining trial blocks $(4-10)$ were also significantly different from Trial Block 1 in the placebo condition. However, during the caffeine session, habituation was not significant until Trial Block 4 (Trials 10-12), demonstrating that caffeine slowed the habituation process. No significant differences were observed between drug conditions with regard to startle

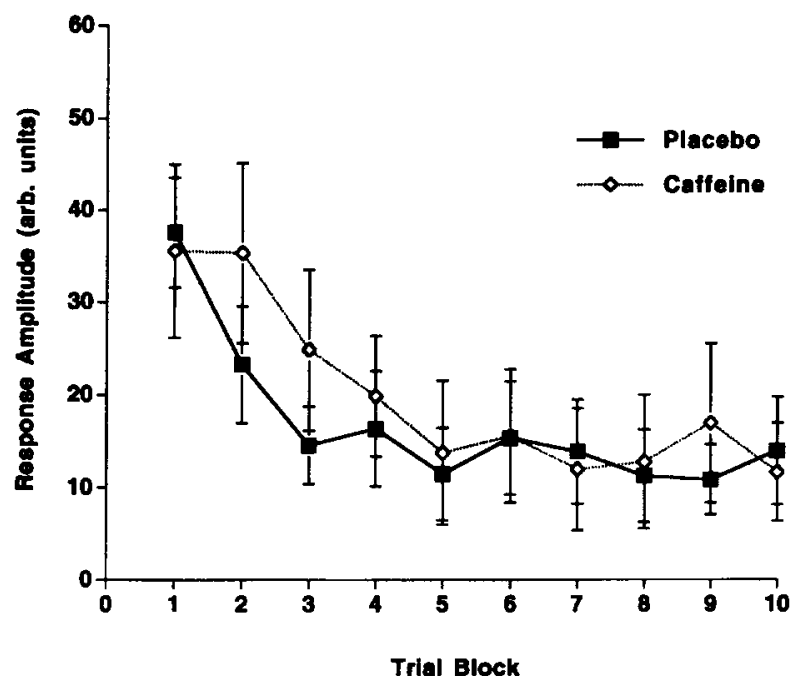

Figure 1. Startle response amplitude as a function of trial blocks in low caffeine users receiving either caffeine or placebo. Vertical bars represent 61 SEM.

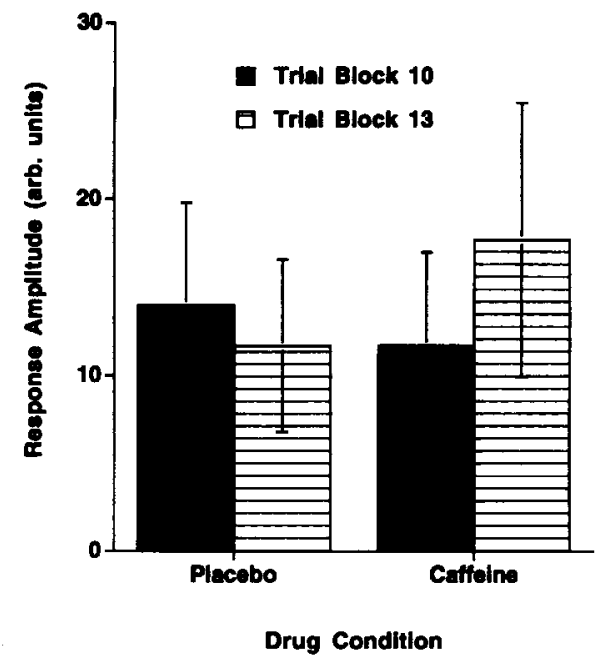

Figure 2. Startle response amplitude in low caffeine users receiving either caffeine or placebo, comparing the last trial block of the habituation phase (Trial Block 10) with the first trial block following the dishabituation phase (Trial Block 13). Vertical bars represent $\pm 1 S E M$.

amplitude on the first trial block, indicating that caffeine did not affect initial startle reactivity.

An orthogonal trend analysis of response latency yielded no significant effects. An orthogonal trend analysis of response probability demonstrated a significant linear trial block effect $[F(1,16)=14.03, p<.01]$, but the interaction of drug $\times$ trial block was not significant. First trial block comparisons did not indicate a significant main effect of drug on response probability.

For the dishabituation analysis, response amplitude and probability on Trial Block 10 were compared with those on Trial Block 13. Dishabituation of response latency was not considered, since no habituation was found for this response measure. A two-way ANOVA of response amplitude revealed a significant trial block $X$ drug interaction $[F(1,16)=7.19, p<.01]$. This interaction illustrated that subjects receiving no caffeine did not dishabituate, whereas, when given caffeine, they dishabituated significantly (Figure 2). No dishabituation was found for response probability.

\section{Discussion}

The findings of Experiment 1 showed that caffeine $(4 \mathrm{mg} / \mathrm{kg})$ delayed startle habituation. It modified startle amplitude habituation selectively, but it did not affect startle probability habituation. This suggests either that these response parameters are subserved by independent neural mechanisms (Blumenthal \& Berg, 1986), or that the habituation of these response parameters is affected differently by caffeine. Startle latency did not habituate, contrary to findings from previous studies (Ornitz \& Guthrie, 1989). Also, significant dishabituation of response amplitude was seen only during the caffeine condition. In Experiment 2, we tested whether caffeine $(4 \mathrm{mg} / \mathrm{kg})$ would exert similar delaying effects on 
startle habituation in high caffeine users. The effects of caffeine withdrawal on startle responding were also studied in Experiment 2.

\section{EXPERIMENT 2}

\section{Method}

Subjects. Ten subjects ( 4 males and 6 females; average age $=$ 18 years, 9 months) participated. They were chosen from an undergraduate introductory psychology class on the basis of their responses to a questionnaire indicating high caffeine intake (i.e., more than $250 \mathrm{mg}$ of caffeine per day; average $=340 \mathrm{mg} /$ day). A caffeine conversion table presented in Avis (1990) was used to translate the subjects' reported daily caffeine intake into milligram values.

Apparatus and Procedure. The stimuli, response measures, materials, apparatus, procedure, and data analysis were the same as in Experiment 1, with the following exception: All 10 subjects were given caffeine $(4 \mathrm{mg} / \mathrm{kg})$ at an initial meeting and either placebo or caffeine $(4 \mathrm{mg} / \mathrm{kg}) 24-26 \mathrm{~h}$ later, at the first testing session. The subjects were asked to abstain from all products containing caffeine during this time interval. Following drug or placebo administration on the 2nd day, startle responses were recorded. One week later, all subjects were again given caffeine during the first visit, and on the 2 nd day they were given the alternate drug condition from the previous week and startle responses were recorded. It should be noted that the subjects were allowed to consume their normal amount of caffeine during the week between sessions. Also, on Day 1 of each week, the subjects received caffeine without being presented with any acoustic startle testing. The purpose of administering caffeine on the 1st day was to ensure that on Day 2, when given placebo or caffeine, subjects would have abstained for exactly $24 \mathrm{~h}$. In this respect, caffeine "withdrawal" was operationally defined as $24 \mathrm{~h}$ without caffeine.

\section{Results}

No significant main effect of drug condition was found for response amplitude, latency, or probability. As in Experiment 1 , an orthogonal trend analysis of response amplitude indicated a significant trial block effect when 10 trial blocks were analyzed $[F(1,18)=45.33, p<.001]$; no significant drug $\times$ trial block interaction was found. Post hoc ANOVAs revealed no significant decrease in response amplitude following Trial Block 5 in the placebo condition or following Trial Block 7 in the caffeine condition, showing that habituation reached asymptote on these trial blocks for the placebo and caffeine conditions, respectively. An orthogonal trend analysis on the first 5 trial blocks revealed a drug $\times$ trial block interaction $[F(1,18)=5.64, p<.05]$. (See Figure 3.)

Specific pairwise comparisons were conducted to assess habituation differences between the caffeine and placebo conditions. Response differences between Trial Blocks 1 and 2 were significantly different in the placebo condition $[F(1,18)=9.44, p<.01]$, but not in the caffeine condition. The remaining trial blocks (3-10) were also significantly different from Trial Block 1 in the placebo condition. However, during the caffeine session, habituation did not occur until Trial Block 5, as was shown through comparison of Trial Blocks 1 and 5 $[F(1,18)=20.14, p<.001]$; this demonstrated that habituation was delayed in the caffeine condition in com-

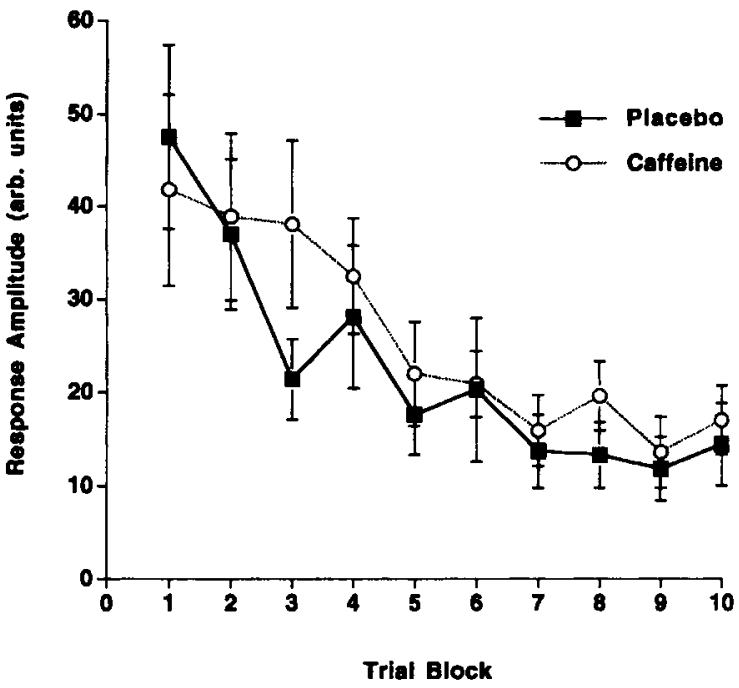

Figure 3. Startle response amplitude as a function of trial blocks in high caffeine users receiving either caffeine or placebo. Vertical bars represent \pm 1 SEM.

parison with the placebo condition (Figure 3). No significant differences were observed between drug conditions with regard to startle amplitude on the first trial block.

An orthogonal trend analysis of response latency revealed no significant effects. An orthogonal trend analysis of response probability demonstrated a significant linear trial block effect $[F(1,18)=7.12, p<.05]$. No significant drug $X$ trial block effect was found for response probability. These findings suggest that habituation of response probability was not affected by caffeine. In contrast to the dishabituation effects observed in Experiment 1 , no significant dishabituation was demonstrated for response amplitude in Experiment 2.

\section{Discussion}

The findings of Experiment 2 show that when high users are given caffeine $(4 \mathrm{mg} / \mathrm{kg})$, habituation is slower than it is when they are given placebo. In support of the results of Experiment 1, caffeine selectively modified startle amplitude habituation, but had no effect on startle probability habituation. Likewise, startle latency did not habituate in Experiment 1 or 2. The lack of dishabituation of response amplitude in Experiment 2 suggests that this phenomenon may be sensitive to an acute dose of caffeine only in persons who do not chronically consume the drug. Although it cannot be proved that the high users had developed caffeine tolerance, it is quite possible that the lack of dishabituation in the high users given caffeine was due to the fact that these persons may have already had some degree of tolerance.

\section{GENERAL DISCUSSION}

The significant trial block effects on response amplitude and probability in both experiments demonstrate 
that these startle responses habituated to acoustic stimuli. The present findings show that caffeine significantly affects habituation of startle response amplitude without having an effect on habituation of startle response probability, and they provide support for the theory that distinct neural mechanisms may underlie these response measures (Blumenthal \& Berg, 1986). It is also possible that these measures differ in their sensitivity to caffeine, but the present research cannot distinguish between these two explanations.

In both experiments, caffeine delayed habituation of acoustic startle reflex amplitude. Caffeine also produced significant dishabituation in Experiment 1, an effect that was not observed in the placebo condition or in either drug condition in Experiment 2. During the dishabituation phase of Experiment 1, the increased responding in the caffeine condition in comparison with the placebo condition may indicate that caffeine enhanced sensitivity to startle in general. However, when response amplitude in the first trial block was compared between the caffeine and placebo conditions, no significant difference between these conditions was demonstrated in Experiment 1 or 2 . Therefore, caffeine did not alter initial startle responding; instead, it affected plasticity of this response.

Another method commonly used to study neuronal plasticity has been the use of the prepulse modification paradigm (Blumenthal \& Levey, 1989; Braff \& Geyer, 1990; Graham, 1975; Hoffman \& Ison, 1980). When a startle eliciting stimulus is preceded by a weak stimulus, the magnitude of the response is altered. We have found that caffeine $(4 \mathrm{mg} / \mathrm{kg})$ produces no significant effect on startle in a prepulse modification paradigm (Blumenthal \& Verma, 1988; Schicatano \& Blumenthal, 1992). These findings indicate that caffeine does not modulate startle plasticity in general; instead, the effects of caffeine on startle are specific to the habituation process.

Neuropharmacologically, caffeine acts as an adenosine antagonist (Snyder, Katims, Annau, Bruns, \& Daly, 1981; Snyder \& Sklar, 1984). Adenosine is a classic neuromodulator in the central nervous system that interacts with a multitude of neurotransmitter systems (Ferre, Fuxe, Euler, Johansson, \& Friedholm, 1992). In view of the fact that adenosine receptors are ubiquitous throughout the central nervous system, adenosine may mediate the normal processing of startle stimuli over time. An acute dose of caffeine in rats increases glucose metabolism in the mesencephalic reticular formation (Nehlig et al., 1986). Since the reticular formation area of the caudal pons is central to the startle reflex circuit (Davis, 1984), this region could be the site for caffeine's effects on starthe habituation. It cannot be ascertained whether this effect is due to direct modulation by adenosine, or whether some other neurotransmitter plays a role.

In the present study, the effects of habitual caffeine use did not differ from acute caffeine effects with regard to startle habituation. It could be argued that the delay of habituation observed in high users was due to with- drawal. The fact that the habituation curves of low and high users in Figures 1 and 3 were similar (i.e., when significant habituation was observed), especially in the placebo conditions, makes this assertion unlikely.

Studying the modulatory effects of caffeine on startle habituation may provide insight into the means by which caffeine acts as a stimulant. Further knowledge of the neuronal (neuroanatomical as well as neurophysiological) relationship between adenosine and other neurotransmitter receptors would help elucidate these stimulant actions at a cellular level. The effects of caffeine on a neurobehavioral process such as habituation supports the tenet that caffeine modulates attention. Warach et al. (1992) asserted that habituation is not a passive decrement in neural activity-that it involves active neural processes. In the startle paradigm, habituation is indicative of an early stage of attentional/sensory filtering. For an individual to pay attention to something, he/she must be able to ignore irrelevant information. In the present experiments, caffeine interrupted this type of filtering in a way that produced persistent responding to redundant sensory information.

\section{REFERENCES}

ANTHONY, B. J. (1985). In the blink of an eye: Implications of reflex modification for information processing. In P. K. Ackles, J. R. Jennings, \& M. G. H. Coles (Eds.), Advances in psychophysiology (Vol.1, pp. 167-218). Greenwich, CT: JAI Press.

Avis, H. (1990). Drugs and life. Dubuque, IA: W. C. Brown.

Blumenthal, T. D. (1988). The startle response to acoustic stimuli near startle threshold: Effects of stimulus rise and fall time, duration, and intensity. Psychophysiology, 25, 607-611.

Blumenthal, T. D., \& BerG, W. K. (1986). Stimulus tise time, intensity, and bandwidth effects on acoustic startle amplitude and probability. Psychophysiology, 23, 635-641.

Blumenthal, T. D., \& GoODE, C. T. (1991). The startle eyeblink response to low intensity acoustic stimuli. Psychophysiology, 28, 296306.

Blumenthal, T. D., \& Levey, B. J. (1989). Prepulse rise time and startle reflex modification: Different effects for discrete and continuous prepulses. Psychophysiology, 26, 158-165.

Blumenthal, T. D., \& Verma, A. (1988). The effects of low levels of caffeine on the human startle reflex. Paper presented at the Southeastern Psychological Association Conference, New Orleans.

BRAFF, D. L., \& GEYER, M. A. (1990). Sensorimotor gating and schizophrenia: Human and animal model studies. Archives of General Psychiatry, 47, 181-188.

Davis, M. (1980). Neurochemical modulation of sensory-motor reactivity: Acoustic and tactile startle reflexes. Neuroscience \& Biobehavioral Reviews, 4, 241-263.

DAvis, M. (1984). The mammalian startle response. In R. C. Eaton (Ed.), Neural mechanisms of startle behavior (pp. 287-351). New York: Plenum.

Davis, M., \& Sheard, M. H. (1974). Habituation and sensitization of the rat startle response: Effects of raphe lesions. Physiology \& Behavior, 12, 425-431.

Ferre, S., Fuxe, K., Euler, G. V., Johansson, B., \& Friedholm, B. B. (1992). Adenosine-dopamine interactions in the brain. Neuroscience, 51, 501-512.

GEYER, M. A., \& BRAFF, D. L. (1987). Startle habituation and sensorimotor gating in schizophrenia and related animal models. Schizophrenia Bulletin, 13, 643-668.

GrAHAM, F. K. (1975). The more or less startling effects of weak prestimulation. Psychophysiology, 12, 238-248.

Griffiths, R. R., Bigelow, G. E., \& Liebson, I. A. (1986). Human cof- 
fee drinking: Reinforcing and physical dependence producing effects of caffeine. Journal of Pharmacology \& Experimental Therapeutics, 239, 416-425.

Griffiths, R. R., \& Woodson, P. P. (1988). Caffeine physical dependence: A review of human and laboratory animal studies. Psychopharmacology, 94, 437-451.

Groves, P. M., \& Thompson, R. F. (1970). Habituation: A dualprocess theory. Psychological Review, 77, 419-459.

HOFFMAN, H. S., \& IsON, J. R. (1980). Reflex modification in the domain of the startle: 1 . Some empirical findings and their implications for how the nervous system processes sensory input. Psychological Review, 87, 175-189.

Lieberman, H. R., Wurtman, R. J., Emde, G. G., Roberts, C., \& Coviela, G. (1987). The effects of low doses of caffeine on human performance and mood. Psychopharmacology, 92, 308-312.

Manspeizer, H. E., \& Blumenthal, T. D. (1986). The effects of small doses of caffeine on the auditory startle response in humans. Psychophysiology, 23, 450. (Abstract)

Nehlig, A., Daval, J. L., Boyet, S., \& VerT, P. (1986). Comparative effects of acute and chronic administration of caffeine on local cerebral glucose utilization in the conscious rat. European Journal of Pharmacology, 129, 93-103.

ORNITZ, E. M., \& GUTHRIE, D. (1989). Long-term habituation and sensitization of the acoustic startle response in the normal adult human. Psychophysiology, 26, 166-173.
Schicatano, E. J., \& Blumenthal, T. D. (1992). [Caffeine does not affect acoustic startle modification]. Unpublished data.

Smith, A. P., Rusted, J. M., Eaton-Williams, P., Savory, M., \& LEATHWOOD, P. (1990). Effects of caffeine given before and after lunch on sustained attention. Neuropsychobiology, 23, 160-163.

Smith, B. D., Rafferty, J., Lindgren, K., Smith, D. A., \& NeSpor, A. (1991). Effects of habitual caffeine use and acute ingestion: Testing a biobehavioral model. Physiology \& Behavior, 51, 131-137.

SNYDER, S. H., Katims, J. J., ANNAU, Z., BRUnS, R. F., \& Daly, J. W. (1981). Adenosine receptors and behavioral actions of methylxanthines. Proceedings of the National Academy of Sciences, 74, $2167-$ 2171 .

SNYDER, S. H., \& SKLAR, P. (1984). Behavioral and molecular actions of caffeine: Focus on adenosine. Journal of Psychiatric Research, 18, 91-106.

WARACh, S., Gur, R. C., Gur, R. E., Skolnick, B. E., Obrist, W. D. \& Reivich, M. (1992). Decreases in frontal and parietal lobe regional cerebral blood flow related to habituation. Journal of Cerebral Blood Flow \& Metabolism, 12, 546-553.

Ward, H. W., Pollare, T., \& GeYER, M. A. (1981). Effects of caffeine on tactile startle in rats. Pharmacology, Biochemistry \& Behavior, $15,875-877$.

(Manuscript received April 21, 1993;

revision accepted for publication October 12, 1993.) 